\title{
Unilateral multicentric cancer of the breast containing two different histopathological types: Case report
}

\author{
TADAHIRO NOZOE ${ }^{1}$, KATSUO SUEISHI ${ }^{2}$, EMIKO MORI $^{1}$, MAYUKO KOHNO $^{1}$, \\ TOMOHIRO IGUCHI ${ }^{1}$, TAKASHI MAEDA ${ }^{1}$ and TAKAHIRO EZAKI ${ }^{1}$ \\ Departments of ${ }^{1}$ Surgery and ${ }^{2}$ Pathology, Fukuoka Higashi Medical Center, Koga 811-3195, Japan
}

Received April 27, 2012; Accepted August 21, 2012

DOI: $10.3892 / 01.2012 .956$

\begin{abstract}
Unilateral multicentric cancer of the breast containing a tumor with a specific histopathological type is comparatively rare. We, herein, report a case of an unilateral multicentric cancer of the breast containing two separate tumors diagnosed as a papillo-tubular carcinoma and an invasive lobular carcinoma. In addition to the difference in histological type, these two tumors also had different patterns of expression of hormonal receptors reflecting the cellular aggressiveness. Therfore, these tumors may be formed through multicentric tumorigenesis.
\end{abstract}

\section{Introduction}

The occurrence of unilateral multiple breast cancer is considered to be derived from multicentric and multifocal tumorigenesis. One theory is that the isolated tumors independently develop from distinctly separate origins and a further theory suggests that the secondary tumor is formed through an intraductal spread of the original tumor. The majority of unilateral multiple breast cancer cases are thought to involve a multifocal tumor derived from an intraductal spread (1).

We, herein, report the case of a post-menopausal female with a unilateral multicentric cancer of the breast containing two isolated tumors with different histopathologic types.

Correspondence to: Dr Tadahiro Nozoe, Department of Surgery, Fukuoka Higashi Medical Center, 1-1-1 Chidori, Koga 811-3195, Japan

E-mail: paper7777@yahoo.co.jp

Key words: breast cancer, multicentric cancer, invasive lobular carcinoma

\section{Case report}

A post-menopausal 78-year-old female presented with a disintegrated tumor in the left breast that had been untreated for one year despite her awareness of the tumor. Physical examination revealed a solid tumor in the internal upper area and a disintegrated tumor in the external lower area of the left breast.

While there was an increase in the white blood cell count in the peripheral blood $\left(18,000 / \mathrm{mm}^{2}\right)$ and serum level of C-reactive protein $(16.2 \mathrm{mg} / \mathrm{dl})$ derived from an inflammatory response to the disintegrated tumor of the breast, there was no increase in the levels of carcinoembryonic antigen (CEA) and cancer antigen 15-3 (CA15-3) in the sera.

Preoperative computed tomography demonstrated two isolated tumors in the left breast (Fig. 1).

The disintegrated tumor was necrotized, emitting a strongly unpleasant smell and bleeding; however, the decision was made to immediately perform a mastectomy.

Macroscopic findings of the specimen revealed two independent tumors measuring $3.5 \mathrm{~cm}$ and $5.0 \mathrm{~cm}$ in length, respectively, that were distinctly separated (Fig. 2).

Histopathological examination revealed a tumor in the internal upper area and a disintegrated tumor in the external lower area of the left breast containing the features of invasive lobular carcinoma and papillo-tubular carcinoma, respectively (Fig. 3). Furthermore, an immunohistochemical examination revealed that the former tumor had positive expression of the estrogen receptor (ER) and progesterone receptor $(\mathrm{PgR})$, but not the HER2 receptor; however, the latter, which comprised the main tumor, expressed none of these receptors (triple negative cancer).

Following mastectomy with an axillary lymph node dissection, radiotherapy (total dose of $50 \mathrm{~Gy}$ ) and adjuvant chemotherapy, consisting of 4 cycles of FEC (5-fluorouracil $500 \mathrm{mg} / \mathrm{m}^{2}$, epirubicin $100 \mathrm{mg} / \mathrm{m}^{2}$ and cyclophosphamide $500 \mathrm{mg} / \mathrm{m}^{2}$ ) every 3 weeks followed by 4 cycles of DOC (docetaxel $75 \mathrm{mg} / \mathrm{m}^{2}$ ) every 3 weeks, were performed. The patient was subsequently treated with an oral intake of tamoxifen in our outpatient clinic and to date, has been in a satisfactory condition without any evidence of tumor recurrence. 


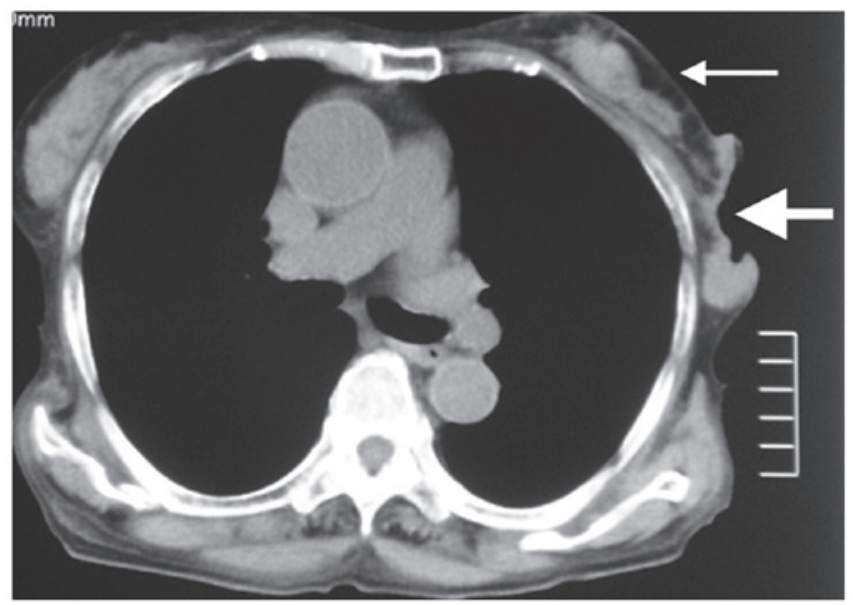

Figure 1. Image of computed tomography. Two isolated tumors (arrows) were revealed in the left breast.

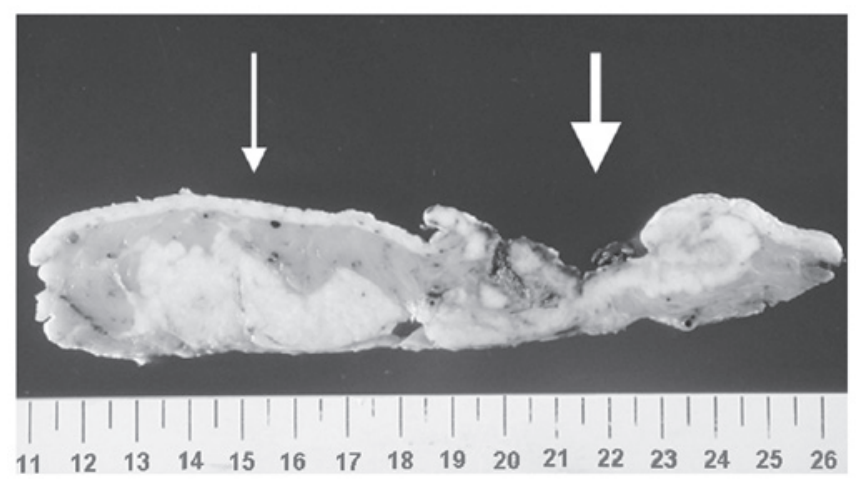

Figure 2. Image of the specimen. Two separate tumors measuring $3.5 \mathrm{~cm}$ and $5.0 \mathrm{~cm}$ in length, respectively, were found (thin and thick arrows). The tumor indicated with a thick arrow is a disintegrated tumor found in the lateral lower area of the breast.

The study was approved by the ethics committee of Fukuoka Higashi Medical Center, Koga, Japan. Informed consent was obtained from the patient.

\section{Discussion}

The incidence of unilateral multiple breast cancer has been reported to account for approximately $5 \%$ of all breast cancers (1). These cases may contain multiple tumors derived from multifocal cancers primarily caused by intraductal spread of the tumor.

According to the criteria presented by Wakabayashi et al (2), unilateral multifocal breast cancer is defined as a histopathologically discontinuous cancer not derived from intraductal spread, containing at least one tumor-free section separating the tumors and involving a large primary tumor and other small secondary tumors.

While the tumors in the present study had different histological types, it was evident that the tumors had been formed by multicentric tumorigenesis.

Unilateral multifocal breast cancer is usually diagnosed following a detailed histological examination of the dissected
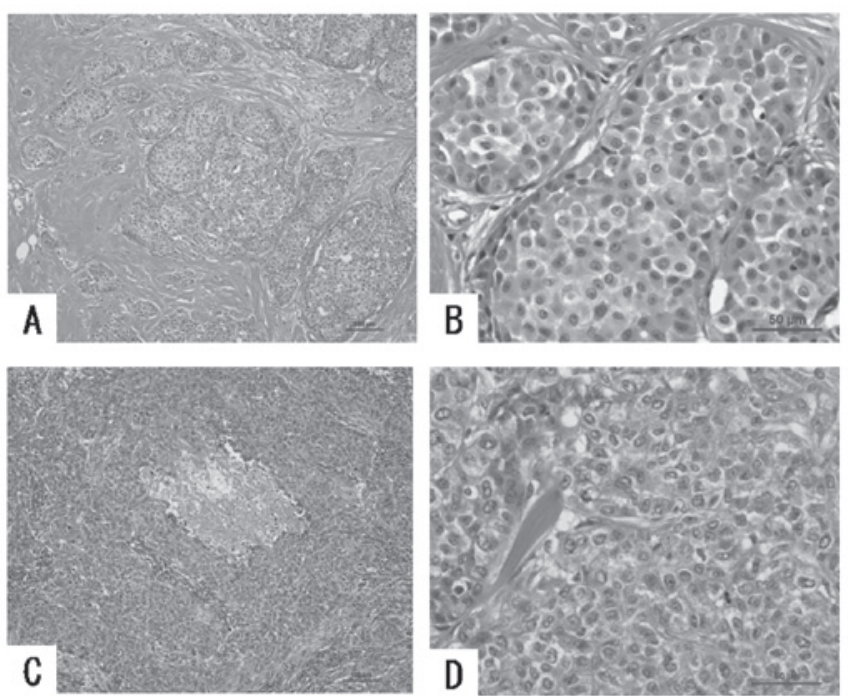

Figure 3. Histopathological findings. The tumor in the internal upper area was diagnosed as invasive lobular carcinoma; (A) lower magnification, (B) higher magnification. The disintegrated tumor in the external lower area was diagnosed as papillo-tubular carcinoma; (C) lower magnification; (D) higher magnification.

specimens. Computed tomography (CT) (3) and magnetic resonance imaging (MRI) (4) have been reported to reveal an unilateral multiple breast cancer prior to surgery, as was demonstrated in our patient, for whom preoperative CT revealed two distinct tumors in the left breast.

In the criteria suggested by Wakabayashi et al (2), there was no reference to the size of the secondary tumors. In the present case, the secondary tumor diagnosed as invasive lobular carcinoma was found to be comparatively smaller than the primary tumor, and its coexistence could only be found by a preoperative physical palpation. The tumor diagnosed as papillo-tubular carcinoma was found to be larger; possibly due to its earlier development. However, although a tumor diagnosed as invasive lobular carcinoma may appear first, a secondary tumor diagnosed as papillo-tubular carcinoma may possess an aggressive behavior caused by the characteristics of triple negative expression (negative for ER, PgR and HER2), causing it to grow rapidly and surpass the other tumor in size.

While the occurrence of multifocal breast cancers has been reported to be more frequent among invasive lobular carcinomas (5), physicians should be cautious not to overlook the secondary tumor in cases of invasive lobular carcinoma of the breast. Moreover, the proportion of tumor recurrence in the abdominal organs including the gastrointestinal tract, ovary and peritoneum has been revealed to be higher in patients who have invasive lobular carcinoma $(6,7)$, and it is well known that tumor recurrence is much more frequent in cases of triple negative breast cancer (8). Therefore, a meticulous follow-up may be mandatory.

In the present study, following mastectomy with an axillary lymph node dissection, radiotherapy and adjuvant chemotherapy, the patient was administered an oral intake of tamoxifen. These treatments may be effective in preventing tumor recurrence. 


\section{References}

1. Koida T, Kimura M, Yanagita Y, et al: Clinicopathological study of unilateral multiple breast cancer. Breast Cancer 8: 202-205, 2001.

2. Wakabayashi T, Tsuchiya SI and Asano G: Unilateral multicentric breast carcinoma studied by whole mammary gland serial sectioning. Breast Cancer 2: 91-98, 1995.

3. Taira N, Ohsumi S, Takabatake D, et al: Contrast-enhanced CT evaluation of clinically and mammographically occult multiple breast tumors in women with unilateral early breast cancer. Jpn J Clin Oncol 38: 419-425, 2008.

4. Sardanelli F, Giuseppetti GM, Panizza P, et al: Italian Trial for Breast MR in Multifocal/Multicentric Cancer. Sensitivity of MRI versus mammography for detecting foci of multifocal, multicentric breast cancer in fatty and dense breasts using the whole-breast pathologic examination as a gold standard. Am J Roentgenol 183: 1149-1157, 2004

5. Dedes KJ and Fink D: Clinical presentation and surgical management of invasive lobular carcinoma of the breast. Breast Dis 30: 31-37, 2009.
6. Arpino G, Bardou VJ, Clark GM, et al: Infiltrating lobular carcinoma of the breast: tumor characteristics and clinical outcome. Breast Cancer Res 6: 149-156, 2004.

7. McLemore EC, Pockaj BA, Reynolds C, et al: Breast cancer: presentation and intervention in women with gastrointestinal metastasis and carcinomatosis. Ann Surg Oncol 12: 886-894, 2005.

8. Nozoe T, Mori E, Iguchi T, et al: Immunohistochemical expression of epidermal growth factor receptor in breast cancer. Breast Cancer 18: 37-41, 2011. 